\title{
Effect of Sulfate-based Cathode-Electrolyte Interphases on Electrochemical Performance of Ni-rich Cathode Material
}

\author{
Bum-Jin Chae ${ }^{1,2, \dagger}$, Hye Ji Song ${ }^{1,2, \dagger}$, Junyoung Mun ${ }^{3 *}$, and Taeeun Yim ${ }^{1,2 *}$ \\ ${ }^{1}$ Department of Chemistry, Incheon National University, 119 Academy-ro, Yeonsu-gu, Incheon 22012, Republic of Korea \\ ${ }^{2}$ Research Institute of Basic Sciences, College of Natural Science, Incheon National University, 119, Academy-ro, Yeonsu-gu, \\ Incheon, 22012, Republic of Korea \\ ${ }^{3}$ Department of Energy and Chemical Engineering, Incheon National University, 119, Academy-ro, Yeonsu-gu, Incheon, \\ 22012, Republic of Korea
}

\begin{abstract}
Recently, layered nickel-rich cathode materials (NCM) have attracted considerable attention as advanced alternative cathode materials for use in lithium-ion batteries (LIBs). However, their inferior surface stability that gives rise to rapid fading of cycling performance is a significant drawback. This paper proposes a simple and convenient coating method that improves the surface stability of NCM using sulfate-based solvents that create artificial cathode-electrolyte interphases (CEI) on the NCM surface. $\mathrm{SO}_{x}$-based artificial CEI layer is successfully coated on the surface of the NCM through a wetcoating process that uses dimethyl sulfone (DMS) and dimethyl sulfoxide (DMSO) as liquid precursors. It is found that the $\mathrm{SO}_{x}$-based artificial CEI layer is well developed on the surface of NCM with a thickness of a few nanometers, and it does not degrade the layered structure of NCM. In cycling performance tests, cells with DMS- or DMSO-modified NCM811 cathodes exhibited improved specific capacity retention at room temperature as well as at high temperature (DMS-NCM811: 99.4\%, DMSO-NCM811: 88.6\%, and NCM811: 78.4\%), as the $\mathrm{SO}_{x}$-based artificial CEI layer effectively suppresses undesired surface reactions such as electrolyte decomposition.
\end{abstract}

Keywords : Lithium Ion Battery, Ni-Rich Cathode, Cathode-Electrolyte Interphases, Surface Stability, Electrochemical Performance

Received: 6 March 2020, Accepted : 18 June 2020

\section{Introduction}

In recent years, researchers have focused on increasing the energy density of energy storage/conversion systems in light of the increasing demand for large-scale applications such as electric vehicles and energy stationary systems [1-3]. As the energy density of lithium-ion batteries (LIBs) is highly dependent on the specific capacity and working potential of the negative and positive electrodes, many attempts have been made to develop advanced electrode materials that satisfy such requirements. Layered lithium

\footnotetext{
${ }^{\dagger}$ This author contributed equally to this work.

*E-mail address: yte0102@inu.ac.kr, jymun@inu.ac.kr DOI: https://doi.org/10.33961/jecst.2020.00829
}

This is an open-access article distributed under the terms of the Creative Commons Attribution Non-Commercial License (http://creativecommons.org/licenses/by-nc/4.0) which permits unrestricted non-commercial use, distribution, and reproduction in an medium, provided the original work is properly cited. nickel-cobalt-manganese oxide $\left(\mathrm{LiNi}_{x} \mathrm{Co}_{y} \mathrm{Mn}_{z} \mathrm{O}_{2}\right.$, $\mathrm{NCM}$ ) is a very promising cathode material owing to its intrinsic physicochemical and electrochemical properties [4-7]. The specific capacity of NCM cathode material is directly proportional to the Ni content in the layered NCM structure as the oxidation potential of $\mathrm{Ni}$ is lower than that of $\mathrm{Co}$, which exhibits high specific capacity under identical cut-off potential of the cell [8-13]. Therefore, the development of NCM cathode materials becomes more important in view of improving the energy density of LIBs.

Despite the aforementioned advantages, NCM cathode materials developed thus far suffer from poor cycling retention. When the $\mathrm{Ni}^{2+}$ in the layered structure is charged, it is converted to $\mathrm{Ni}^{4+}$ by the electrochemical reaction. At this stage, the extremely unstable $\mathrm{Ni}^{4+}$ species tends to return to the more thermodynamically stable $\mathrm{Ni}^{3+}$ or $\mathrm{Ni}^{2+}$ species. Hence, the electron-rich electrolyte can be easily decom- 
(a)

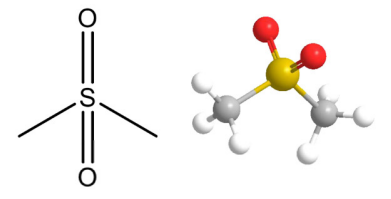

DMS

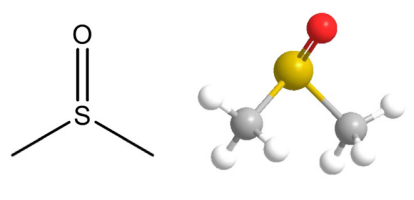

DMSO

(b)

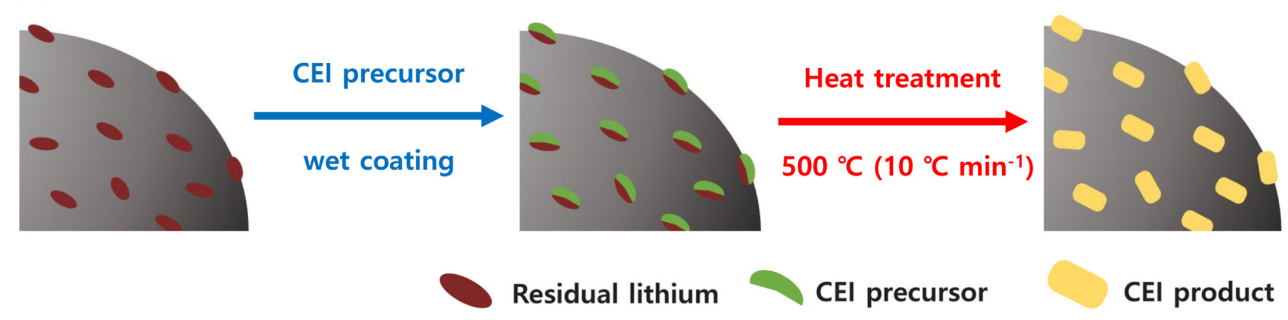

Fig. 1. (a) 2D- and 3D-molecular structure of DMS and DMSO, and (b) scheme for synthesis of surface-modified NCM811 cathode materials.

posed at the electrode/electrolyte interface because it can easily donate non-bonding electrons to the unstable $\mathrm{Ni}^{4+}$ species, resulting in electrolyte decomposition at the interface [14-18]. Even though increasing the Ni content in the layered NCM structure can augment the specific capacity, it also accelerates electrolyte decomposition in the cell, leading to poor cycling performance. Therefore, this trade-off relationship should be optimized in the NCM in order to simultaneously satisfy the high energy density requirements together with longer cycling performance of LIBs.

In this context, here we proposed a simple and convenient approach using a sulfate-based solvent that effectively improves the surface stability of NCM cathode material (Fig. 1). Our concept is based on embedding a sulfonate $\left(\mathrm{SO}_{x}\right)$-based artificial cathodeelectrolyte interphase (CEI) layer on the NCM cathode material by a single-step thermal treatment process. It has been well documented that $\mathrm{SO}_{x}$-based CEI layers are effective for suppressing electrolyte decomposition at the electrode/electrolyte interface [19-27]. As the $\mathrm{SO}_{x}$-based CEI layers conduct ions but are electronic insulators, they do not allow electron transfer reaction between the electrode material and electrolyte, thereby improving the surface stability during the electrochemical charging/discharging process. In other words, the partially localized charge state of $\mathrm{SO}_{x}$-based CEI layers $\left(\mathrm{S}^{+}-\mathrm{O}^{-}\right)$allows $\mathrm{Li}^{+}$ migration at the electrode/electrolyte interface under electrochemical polarization, but it protects the $\mathrm{Ni}^{4+}$ species in the cell, leading to the retention of high specific capacity even after several cycles.

The proposed approach for the surface modification of the NCM is comprised of 1) wet-coating of NCM with coating precursor followed by 2) thermal treatment of recovered precipitants. Dimethyl sulfone (DMS) and dimethyl sulfoxide (DMSO) that have sulfonyl functional groups are selected as precursors for the $\mathrm{SO}_{x}$-based CEI layers. As DMS and DMSO are common solvents in the chemical industry, indicating that this process can be inexpensively realized. In addition, both the precursors are liquids, making it easy to recover used precursors in the wet-coating process by simple filtration. It also indicates that the suggested approach could be scalable in terms of processability. Besides, the liquid-based surface modification is also expected to reduce residual lithium species such as lithium hydroxide $(\mathrm{LiOH})$ and lithium carbonate $\left(\mathrm{Li}_{2} \mathrm{CO}_{3}\right)$ on the NCM cathode, which may cause serious swelling of the electrode during operation due to the irreversible electrochemical decomposition of such residual lithium species. The presence of a certain amount of residual lithium species (used in the synthetic step for NCM cathode material) on the NCM cathode is unavoidable because calcination temperature for NCM cathode material is higher than the NCM cathode has a low 
Ni composition $[28,29]$. In this respect, the wet-coating process using liquid precursors (DMS and DMSO) would be an effective way for decreasing residual lithium species on NCM cathodes as those can be washed off in the filtration stage itself. Using these strategies, we modify the surface state of the NCM by employing sulfonyl group functionalized task-specific precursors, and the electrochemical properties of the resulting material are systematically characterized.

\section{Experimental}

\subsection{Typical procedure for wet-coating and heat} treatment process

For wet-coating of $\mathrm{LiNi}_{0.8} \mathrm{Co}_{0.1} \mathrm{Mn}_{0.1} \mathrm{O}_{2}(\mathrm{NCM} 811)$, $2.0 \mathrm{~mL}$ of dimethyl sulfone (Aldrich) or $2.0 \mathrm{~mL}$ of dimethyl sulfoxide (Aldrich) was dissolved in $10.0 \mathrm{~mL}$ of $\mathrm{N}$-methyl pyrrolidone (Aldrich) to prepare the coating solution (all reagents were used as received). Then, $10.0 \mathrm{~g}$ of NCM811 was added to the prepared coating solution and it was subjected to stirring for $10 \mathrm{~min}$. Wet-coated NCM811 was then separated by filtration and thereafter, the collected $\mathrm{NCM} 811$ was heated to $500^{\circ} \mathrm{C}$ at a rate of $10^{\circ} \mathrm{C} \mathrm{min}$. When the temperature reached $500^{\circ} \mathrm{C}$, it was held at the same temperature for $3 \mathrm{~h}$, subsequent to which the temperature was lowered to $25^{\circ} \mathrm{C}$ at a rate of $10^{\circ} \mathrm{C}$ $\mathrm{min}^{-1}$. The surface morphologies of DMS- and DMSO-modified NCM811 were characterized by field-emission scanning electron microscope (FESEM, JSM-7001F, JEOL) and transmission electron microscope (TEM, TALOS F200X, FEI). The bulk structures of DMS- and DMSO-modified NCM811 were examined by x-ray diffraction (XRD, SmartLab, Rigaku) equipped with monochromatic $\mathrm{Cu} \mathrm{K} \alpha$ radiation $(\lambda=1.54056 \AA)$ and their chemical compositions were analyzed by Fourier-transform infrared spectroscopy (FT-IR, VERTEX $80 \mathrm{~V}$, Bruker) in the attenuated total reflectance mode, and $\mathrm{X}$-ray photoelectron spectroscopy (XPS, K $\alpha$, PHI 5000 versa Probe II) under $\mathrm{N}_{2}$ atmosphere.

\subsection{Electrode preparation and evaluating cycling performances}

NCM811 cathodes were prepared as follows. A mixture of NCM811 (or modified NCM811), poly(vinylidene fluoride) (PVDF) (KF3000, Kureha), and carbon black (Super P) with a ratio of 96:2:2 (weight \%, 2.00 g of NCM811) was well dis- persed in $N$-methyl pyrrolidone at least $1 \mathrm{~h}$ to prepare the cathode slurry. The resulting slurry was then coated on aluminum foil and dried overnight in a vacuum oven at $120{ }^{\circ} \mathrm{C}$ for overnight. The loading density of the cathode was $11.75 \pm 0.75 \mathrm{mg} \mathrm{cm}^{-2}$. To evaluate cycling performance, 2032 coin-cells were fabricated with a cathode (NCM811 or modified NCM811), a Li-metal anode, a poly(ethylene) (PE) separator, and electrolytes $(\mathrm{EC}: \mathrm{EMC}=1: 2+1 M$ $\mathrm{LiPF}_{6}$, PanaxEtec). The cells were charged to $4.25 \mathrm{~V}$ (vs. $\mathrm{Li} / \mathrm{Li}^{+}$) by constant current-constant voltage mode and discharged to $3.00 \mathrm{~V}\left(\mathrm{vs} . \mathrm{Li} / \mathrm{Li}^{+}\right.$) by constant current mode with a $0.1 \mathrm{C}\left(18 \mathrm{~mA} \mathrm{~g}^{-1}\right)$ current for two cycles (the formation step), following which they were charged/discharged with a $1.0 \mathrm{C}$ current $\left(180 \mathrm{~mA} \mathrm{~g}^{-1}\right)$ for 50 cycles at room temperature or at $45^{\circ} \mathrm{C}$ on a charge/discharge cycler (WBCS3000, Wonatech). After completion of the cycling, each cell was disassembled in a glove box in order to recover the cycled NCM811 cathode, which was quickly washed using dimethyl carbonate. The surface morphology of the recovered NCM811 cathodes was examined using SEM.

\section{Results and Discussion}

Surface morphologies of DMS and DMSO-treated NCM811 cathode materials were characterized by SEM and TEM analyses (Fig. 2). Interestingly, DMS and DMSO-treated NCM811 cathode materials showed different surface morphologies while the structures of the liquid precursors used for surface modification are almost identical. This may be attributed to the difference in chemical reactivity between DMS and DMSO: it can be purported that DMS covers the CEI layer more efficiently by binding with other reactive species on the electrode surface. The analysis of residual lithium species on the NCM811 cathode material supported this explanation. Before the surface modification of NCM811 cathode material, the total amount of residual lithium species was 18,863 ppm ( $\mathrm{LiOH}: 13,022 \mathrm{ppm}$ and $\mathrm{Li}_{2-}$ $\left.\mathrm{CO}_{3}: 5,841\right)$. After surface modification of it, this value was considerably reduced: $9,132 \mathrm{ppm}$ for DMS-treated NCM811 and 12,017 ppm for DMSOtreated NCM811. This indicated that the proposed approach involving DMS- and DMSO-based treatment changes the surface state of NCM811 by using the residual lithium species as seeds for CEI growth. 


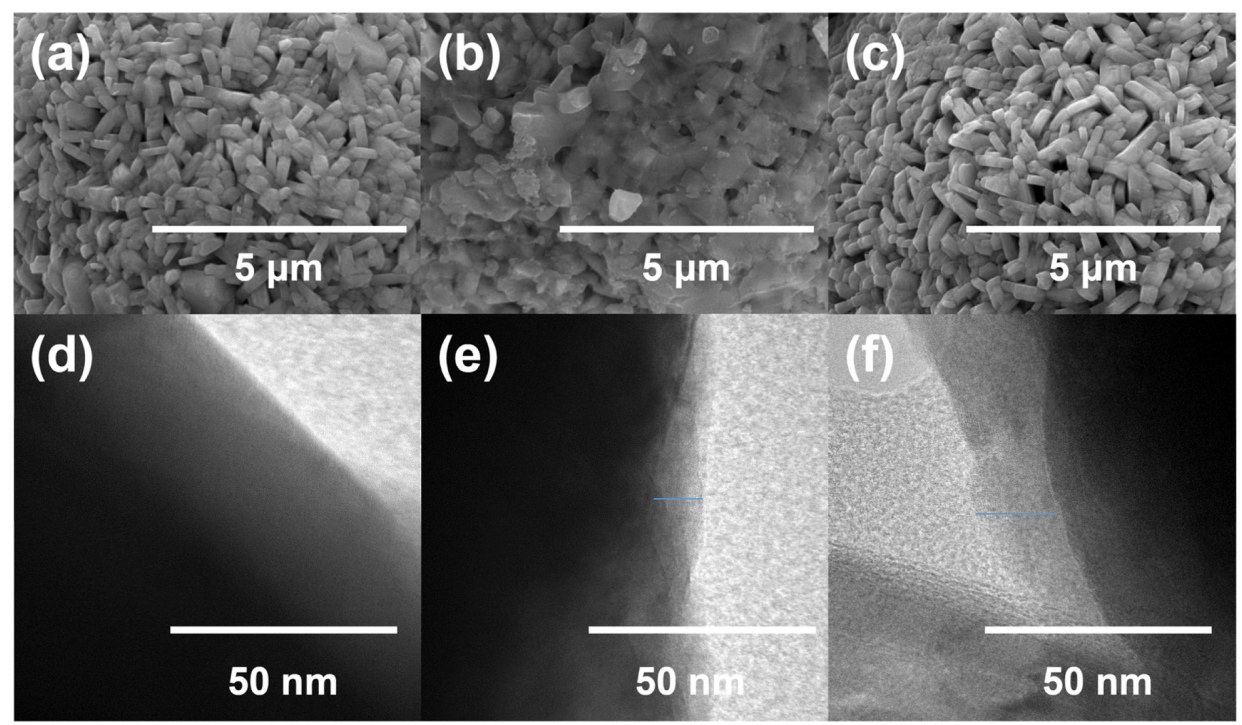

Fig. 2. SEM images for (a) bare NCM811, (b) DMS-modified NCM811, and (c) DMSO-modified NCM811. TEM images for (d) bare NCM811, (e) DMS-modified NCM811, and (f) DMSO-modified NCM811.

(a)

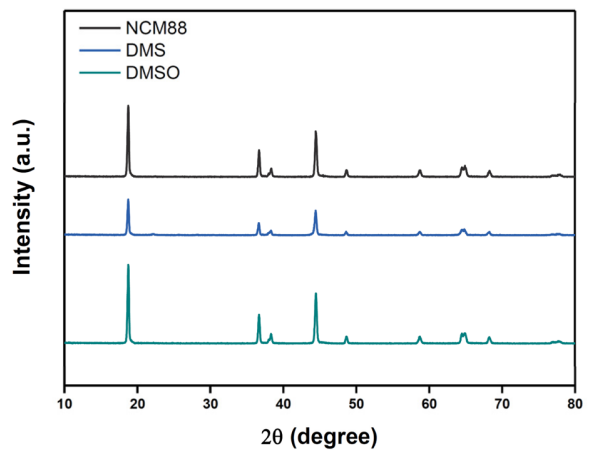

(b)

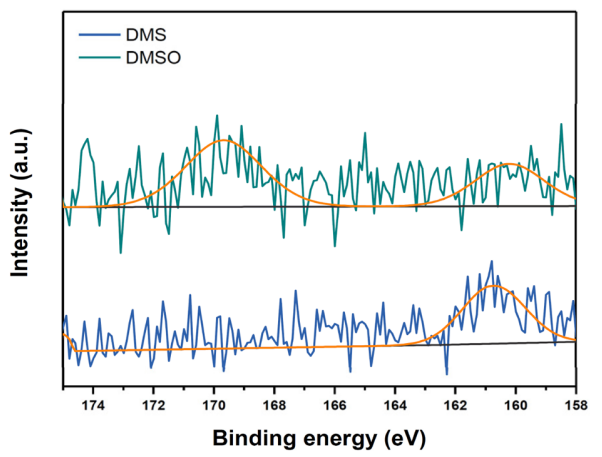

Fig. 3. (a) XRD and (b) S2p XPS analysis results (black: bare NCM811, blue: DMS-modified NCM811, and green: DMSOmodified NCM811).

In particular, DMS-treated NCM811 showed a remarkable decrease in residual lithium species after surface modification, indicating that modification precursors that have high reactivity provide better CEI coverage of the NCM811 cathode material. The results of the electron dispersive spectroscopy (EDS) analysis support this conclusion: $4.59 \%$ of $\mathrm{S}$ was found in DMS-treated NCM811, while $0.33 \%$ of S was observed in DMSO-treated NCM811. Although there were local differences, the thickness of the CEI layer on the surface of NCM811 was approximately
$10 \mathrm{~nm}$ for DMS-treated NCM811 and $16 \mathrm{~nm}$ for DMSO-treated NCM811 as confirmed by TEM.

To verify effect of surface modification by $\mathrm{SO}_{x}$ based liquid precursors on their bulk and surface properties, modified NCM811 cathode materials were analyzed by XRD (Fig. 3a). As all the intrinsic peaks associated with the layered structure were observed for both DMS- and DMSO-modified NCM811 cathode, it could be concluded that the proposed method does not affect the bulk properties of NCM811, but only changes the surface properties. In 
the XPS analysis for S2p spectra (Figure $3 \mathrm{~b}$ ), $\mathrm{SO}_{x}$ peak indicating the low oxidation state of $\mathrm{S}$ was found at $160.7 \mathrm{eV}$ [30] for DMS-modified NCM811 and the $\mathrm{SO}_{x}$ peak associated with high oxidation state of S was observed at $169.7 \mathrm{eV}[31,32]$ for DMSOmodified NCM811. This result indicates that the surface of NCM811 was modified by $\mathrm{SO}_{x}$-based liquid precursors.

Based on the results obtained from material characterization studies, the electrochemical performances of the candidate materials were evaluated. Roomtemperature cycling test results indicated that surface modification using $\mathrm{SO}_{x}$-based liquid precursors was effective (Fig. 4a). Although the cells with DMS- and DMSO-modified NCM811 showed slightly low discharge specific capacities initially owing to the additional CEI layer on their surfaces $\left(194.4 \mathrm{~mA} \mathrm{~h} \mathrm{~g}^{-1}\right.$ for bare NCM811, 181.2 $\mathrm{mA} \mathrm{h} \mathrm{g}^{-1}$ for DMS-modified NCM811 and $193.3 \mathrm{~mA} \mathrm{~h} \mathrm{~g}^{-1}$ for DMSO-modified NCM811), their initial coulombic efficiencies were high compared with bare NCM811 (85.2\% for bare NCM811, 88.5\% for DMS-modified NCM811, and

(a)

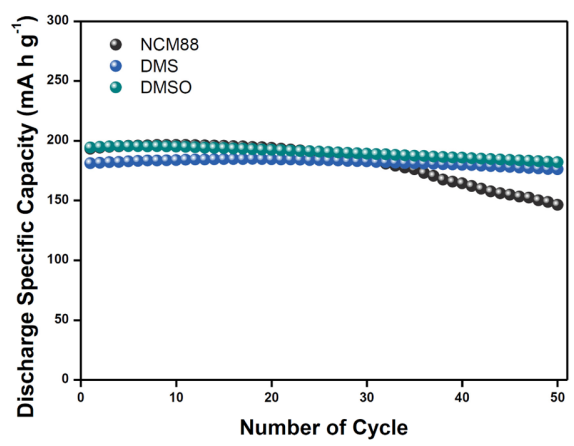

$87.0 \%$ for DMSO-modified NCM811). This means that the artificially induced $\mathrm{SO}_{x}$-based CEI layer was effective in suppressing undesired surface reactions such as the electrolyte decomposition reaction [20,23,25-27]. Thus, cycling retention of the cells with DMS- and DMSO-modified NCM811 cathodes was improved compared with the bare NCM811 cathode: DMS- and DMSO-modified NCM811 showed $97.2 \%$ and $93.7 \%$ specific capacity retention while the bare NCM811 retained $75.7 \%$ of its specific capacity after 50 cycles. During high temperature cycling, modified NCM811 cathode materials exhibited improved cycling retention (Fig. 4b). The cell with bare NCM811 showed drastic fading of cycling performance, dropping down to $78.4 \%$ of cycling retention after 50 cycles. This is typical of NCMs because undesired surface reactions including electrolyte decomposition frequently occur on the unstable surface of the cathode, leading to the drastic decrease in cycling retention $[33,34]$. On the other hand, the cells with DMS- and DMSO-modified NCM811 cathodes exhibited much improved cycling

Fig. 4. Cycling performances at (a) room temperature, and (b) high temperature (black: bare NCM811, blue: DMSmodified NCM811, and green: DMSO-modified NCM811).

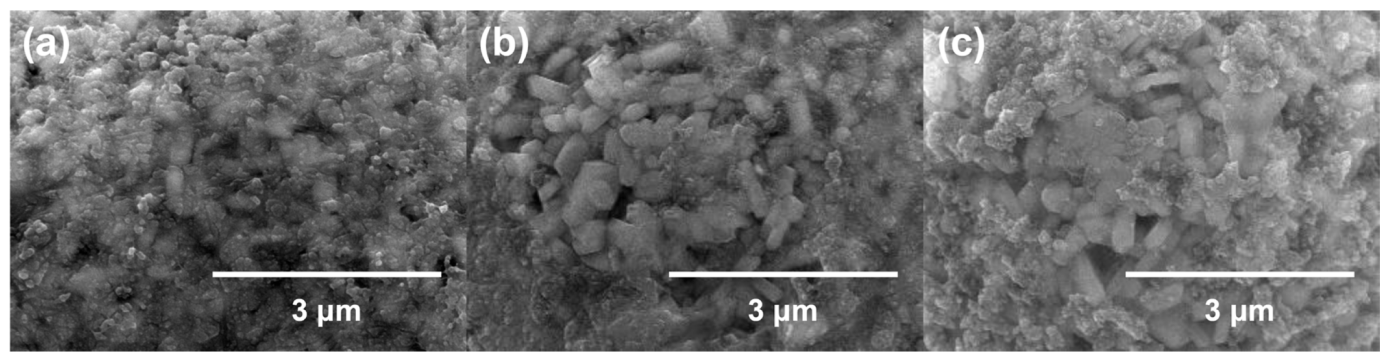

Fig. 5. SEM images for cycled (a) bare NCM811, (b) DMS-modified NCM811, and (c) DMSO-modified NCM811. 
behavior: $88.6 \%$ and $99.4 \%$ of their respective discharge specific capacities were retained after 50 cycles. Additional SEM analysis for recovered cycled NCM811 cathodes provided informative clues regarding the role played by the artificially induced $\mathrm{SO}_{x}$-based CEI layer during electrochemical cycling (Fig. 5). It was easy to demarcate the secondary particles for recovered DMS- and DMSO-modified NCM811, while, post-cycling, the bare NCM811 seemed to be covered entirely with decomposed adducts attributed to electrolyte decomposition. It means that the NCM811 cathode was continually affected by poor interfacial stability during electrochemical cycling while DMS- and DMSO-modified NCM811 cathodes were free from such electrolyte decomposition. This indicates that the simple and convenient wet-coating-based surface modification approach is effective not only for limiting the presence of residual lithium species on the NCM811 cathode, but also for improving the surface stability of the cathode, which can, ultimately, result in better safety and electrochemical performance of LIBs.

\section{Conclusions}

To improve the surface stability of NCM811 cathode materials, an $\mathrm{SO}_{x}$-based artificial CEI layer was embedded on the surface of the NCM811 cathode using DMS and DMSO as liquid precursors. It was confirmed by SEM and TEM analyses that the $\mathrm{SO}_{x^{-}}$ based artificial CEI layer was well developed on the NCM811 cathode surface. The images showed that a few nanometer-thick CEI layer was finely coated on the surface of the NCM811 cathode. XRD analysis revealed that the newly proposed coating approach did not affect the bulk structure of NCM811 (layered structure), while XPS studies indicated that the artificial CEI layer was composed of $\mathrm{SO}_{x}$-functional groups that effectively suppress undesired surface reactions like electrolyte decomposition. As a result, the cells equipped with DMS- or DMSO-modified NCM811 cathodes exhibited improved specific capacity retentions at room temperature and elevated temperature (DMS-NCM811: 99.4\%, DMSONCM811: 88.6\%, and NCM811: 78.4\%) after 50 cycles. We believe that the proposed approach is useful in improving the surface stability of NCM because it can achieve desirable results by a simple, inexpensive, and convenient one-step process.

\section{Acknowledgment}

This work was supported by Incheon National University Research Grant in 2018.

\section{References}

[1] L. Lu, X. Han, J. Li, J. Hua, M. Ouyang, J. Power Sources 2013, 226, 272-288.

[2] M. M. Thackeray, C. Wolverton, E. D. Isaacs, Energy Environ. Sci. 2012, 5, 7854-7863.

[3] E. Karden, S. Ploumen, B. Fricke, T. Miller, K. Snyder, J. Power Sources 2007, 168(1), 2-11.

[4] J. Zheng, W. H. Kan, A. Manthiram, ACS Appl. Mater. Interfaces 2015, 7(12), 6926-6934.

[5] N. Yabuuchi, T. Ohzuku, J. Power Sources 2003, 119, 171-174.

[6] S. Liu, L. Xiong, C. He, J. Power Sources 2014, 261, 285-291.

[7] Y. Chung, H.-Y. Park, S.-H. Oh, D. Y. Yoon, S.-W. Jin, D.-Y. Jang, J. M. Ko, W. I. Cho, S.-R. Lee, J. Electroceramics, 2013, 31(3-4), 316-323.

[8] K. Kang, Y. S. Meng, J. Breger, C. P. Grey, G. Cender, Science 2006, 311(5763), 977-980.

[9] C. M. Julien, A. Mauger, K. Zaghib, H. Groult, Inorganics 2014, 2(1), 132-154.

[10] D. D. MacNeil, Z. Lu, J. R. Dahn, J. Electrochem. Soc. 2002, 149(10), A1332-A1336.

[11] C.-C. Wang, A. Manthiram, J. Mater. Chem. A 2013, 1(35), 10209-10217.

[12] J. Ahn, J. H. Kim, B. W. Cho, K. Y. Chung, S. Kim, J. W Choi, S. H. Oh, Nano Lett. 2017, 17(12), 7869-7877.

[13] H. J. Song, S. H. Jang, J. Ahn, S. H. Oh, T. Yim, J. Power Sources 2019, 416, 1-8.

[14] J.-Y. Hwang, C. S. Yoon, I. Belharouak, Y.-K. Sun, J. Mater. Chem. A 2016, 4(46), 17952-17959.

[15] Y. K. Sun, Z. Chen, H. J. Noh, D. J. Lee, H. G. Jung, Y. Ren, S. Wang, C. S. Yoon, S. T. Myung, K. Amine, Nat. Mater. 2012, 11(11), 942-947.

[16] W. Liu, P. Oh, X. Liu, M.-J. Lee, W. Cho, S. Chae, Y. Kim, J. Cho, Angew. Chem. Int. Ed. 2015, 54(15), 44404457.

[17] M. Dixit, B. Markovsky, F. Schipper, D. Aurbach, D. T. Major, J. Phys. Chem. C 2017, 121(41), 22628-22636.

[18] S. H. Jang, J. Mun, D.-K. Kang, T. Yim, J. Electrochem. Sci. Technol. 2017, 8(2), 162-168.

[19] B.-J. Chae, T. Yim, J. Power Sources 2017, 360, 480487.

[20] S. H. Lim, W. Cho, Y.-J. Kim, T. Yim, J. Power Sources 2016, 336, 465-474.

[21] B.-J. Chae, T. Yim, Mater. Chem. Phys. 2018, 214, 6672.

[22] B. Zhang, M. Metzger, S. Solchenbach, M. Payne, S. Meini, H. A. Gasteiger, A. Garsuch, B. L. Lucht, $J$. Phys. Chem. C 2015, 119(21), 11337-11348. 
[23] J. Pires, L. Timperman, A. Castets, J. S. Pena, E. Dumont, S. Levasseur, R. Dedryvere, M. Anouti, RSC $A d v$. 2015, 5(52), 42088-42094.

[24] G. H. Wrodnigg, T. M. Wrodnigg, J. O. Besenhard, M. Winter, Electrochemistry Communications 1999, 1(3-4), 148-150.

[25] G. H. Wrodnigg, J. O. Besenhard, M. Winter, J. Electrochem. Soc. 1999, 146(2), 470-472.

[26] K. J. Nelson, J. Xia, J. R. Dahn. J. Electrochem. Soc. 2014, 161(12), A1884-A1889.

[27] J. Xia, J. E. Harlow, R. Petibon, J. C. Burns, L. P. Chen, J. R. Dahn, J. Electrochem. Soc. 2014, 161(4), A547A553.

[28] J. Choi, A. Manthiram, Electrochem. Solid-State Lett. 2005, 8(8), C102-C105.
[29] H.-J. Noh, S. Youn, C. S. Yoon, Y.-K. Sun, J. Power Sources 2013, 233, 121-130.

[30] A. Calborean, F. Martin, D. Marconi, R. Turcu, I. E. Kacso, L. Buimaga-Iarinca, F. Graur, I. Turcu, PMater. Sci. Eng. C. 2015, 57, 171-180.

[31] J. Baltrusaitis, D. M. Cwiertny, V. H. Grassian, Phys. Chem. Chem. Phys. 2007, 9(41), 5542-5554.

[32] Z. Huang, Q. Lu, J. Wang, X. Chen, X. Mao, Z. He, PLoS One 2017, 12(8), e0183617.

[33] J. Wang, Y. Yu, B. Li, T. Fu, D. Xie, J. Cai, J. Zhao, Phys. Chem. Chem. Phys. 2015, 17(47), 32033-32043.

[34] D. R. Gallus, R. Schmitz, R. Wagner, B. Hoffmann, S. Nowak, I. Cekic-Laskovic, R. W. Schmitz, M. Winter, Electrochim. Acta 2014, 134, 393-398. 